\title{
The $E_{7}$ Commuting Squares Produce $D_{10}$ as Principal Graph
}

\author{
By
}

David E. Evans* and Yasuyuki Kawahigashi**

\begin{abstract}
We prove that the (two) connections, or commuting squares, on the Coxeter-Dynkin diagram $E_{7}$ produce a subfactor with principal graph $D_{10}$. This was conjectured by J.-B. Zuber in connection with modular invariants in conformal field theory, and solve the last case of computing the flat parts of the connections on Coxeter-Dynkin diagrams with index less than 4 .
\end{abstract}

\section{§0. Introduction}

Since V.F.R. Jones initiated a systematic study of subfactors in [Jo], more and more connections of the subfactor theory with topology and quantum field theory have been pointed out. Our aim in this paper is to provide an evidence of a deeper relation between a notion of flatness in subfactor theory and modular invariants in conformal field theory through a computation of flat parts of a commuting square arising from the Coxeter-Dynkin diagram $E_{7}$, which was the last case left among the $A-D-E$ diagrams.

In theory of operator algebras, a classification problem of subfactors (of the approximately finite dimensional factor of type $\mathrm{II}_{1}$ ) have attracted much attention, and approach using higher relative commutants has been quite successful $[\mathbf{O} 1, \mathbb{P} 2, \mathbf{P} 3]$. Ocneanu's paragroup machinery $[\mathbf{O} 1, \mathbf{0 3}]$ appeared as a combinatorial characterization of higher relative commutants and it has a strong resemblance to some combinatorial aspects of statistical mechanics and rational conformal field theory such as $[\mathbf{A B F}, \mathbf{B a}, \mathbf{D H V W}, \mathbf{D J M O}, \mathbf{D Z} 1$, $\mathbf{D Z 2}, \mathbf{D V}, \mathbf{F}, \mathbf{F G}, \mathbf{K o}$, So, $\mathbb{Z}, \mathbf{W}]$, as noted and exploited in $[\mathbf{B G}, \mathbb{E K} 1, \mathbf{E K} 2$,

Communicated by H. Araki, April 7, 1993.

1991 Mathematics Subject Classification: 46L37

* Department of Mathematics and Computer Science, University College of Swansea, Singleton Park, Swansea SA28PP, Wales, U.K.

** Department of Mathematical Sciences, University of Tokyo, Hongo, Tokyo, 113, Japan 
$\mathbb{I}, \mathbb{K}$ I $, \mathbb{K} a 2, \mathbb{P a}, \mathbb{R}]$. For example, the commuting square condition in subfactor theory $[\mathbb{P} \mathbb{1}, \mathbb{G H}, \mathbb{S c}]$ is related to the crossing symmetry or second inversion relation in solvable lattice model theory. (See $[\mathbb{A} \mathbb{E}, \mathbb{B}, \mathbb{D} \mathbb{M}$, 耳i] for more on solvable lattice models.)

The subfactors of the approximately finite dimensional (AFD) factor of type $\mathbb{I I}_{1}$ with index less than 4 have attracted much attention. In these cases, the so-called principal graph of the inclusion must be one of the Coxeter-Dynkin diagrams of type $A, D, E$, as observed in [ $G H]$, $\S 4.1$. (Each graph has a distinguished vertex denoted by $*$, determined as the vertex with the least entry of the Perron-Frobenius eigenvector. See [P2, Theorem 3.8], [I1].) Then for each graph, we have an additional structure - a "connection" giving a commuting square. The commuting squares can be written down explicitly as in [GHI], §4.2] for the $A-D-E$ graphs, and all the connections can be classified as follows $[\mathrm{O}, 03, \mathrm{~K} \Omega \mathbb{1}$ ]: Each of the diagrams of type $A$ or $D$ has a unique connection, and each of the diagrams of type $E$ has two connections. The remaining problem is to decide whether they really appear as higher relative commutants of subfactors. Alternatively, in Popa's terminology [ $\mathbb{P} 2]$, we have to determine whether or not these commuting squares are canonical commuting squares. Ocneanu claimed that this question can in general be solved by checking finitely many explicit equations, and when these are satisfied the connection is said to be flat $[\mathrm{O} \mathbb{1}, \mathbb{O}, \mathbb{K a l}, \mathbb{K} 22]$. This flatness is a key axiom in Ocneanu's combinatorial approach, and it also plays a key role in construction of topological invariants of 3-manifolds of type Turaev-Viro $[\mathbb{T V}, \mathbb{D} J \mathbb{N}]$ from subfactors $[04, \mathbb{E} 2]$. In the case of $A-D-E$ diagrams, Ocneanu announced the following claim [O1]: The connections on $A_{n}, D_{2 n}$, $E_{6}, E_{8}$ are flat, but those on $D_{2 n+1}, E_{7}$ are not flat. $\mathrm{He}$ has not shown full details of his method, but these claims have been proven by several people [H1, $\mathbb{1} 2, \mathbb{K a l}, \mathrm{SV}]$. (The claim for $A_{n}$ is easy to verify, and $E_{6}$ has been realized by [BN]. Impossibility of $E_{7}$ and $D_{5}$ is implicit in [P2, Theorem 3.8].) The classification is thus complete for index less than four, but it is natural to ask what happens in the case of non-flat connection. We have commuting squares even for non-flat connections and hence subfactors. So we have a problem of determining the principal graph. In Ocneanu's terminology [03], this is the problem of determining a flat part for a non-flat connection.

In the case of $D_{2 n+1}$, we can conclude except for the $D_{7}$ case that the principal graph is $A_{4 n-1}$ by considering only the value of the Jones 
index. The index value $4 \cos ^{2} \frac{\pi}{12}$ for $D_{7}$ is realized for $A_{11}$ and $E_{6}$, but by the compactness argument of Ocneanu [O3, II.6], we know that the higher relative commutants of this subfactor are contained in the string algebra of $D_{7}$, which eliminates the possibility of $E_{6}$. The case of $E_{7}$, however, is more subtle. By looking at the index value, we know that the principal graph is either $D_{10}$ or $A_{17}$, but it seems that there is no easy way to determine which is the right one. Our object in this paper is to prove that $D_{10}$ is the correct invariant. As far as we know, this is the first example of a direct computation of a flat part of the non-flat connection. Our method comes from Izumi's paper [12] on the flatness of the $E_{8}$ connections.

Cappelli, Itzykson and Zuber $[\mathrm{CIZ}]$ and A. Kato $[\mathbb{K} \mathbf{t}]$ encountered an $A-D-E$ classification in conformal field theory, and Zuber noticed a strong similarity to the subfactor classification mentioned above. Based on this observation, Zuber conjectured that the $E_{7}$ connections produce the $D_{10}$ principal graph. Our result here proves this conjecture, and provides the last missing piece in similarity between the $A-D-E$ classifications in subfactor theory and solvable lattice model theory.

After this work was completed, we learned that $U$. Haagerup independently and recently obtained the same result with the essentially same method.

This work was completed while the first author visited MSRI, Berkeley in the spring of 1992. We wish to thank MSRI and Irving Kaplansky for the financial support and their hospitality, D. Bisch for informing us of Haagerup's result, U. Haagerup for explaining his method, which turned out to be the same as ours, J.-B. Zuber for making the conjecture during a visit to Swansea in February 1992, and the London Mathematical Society for financial support for making that visit possible. The second author was supported by a Miller Research Fellowship at University of California, Berkeley.

\section{§1. Embedding of Path Algebras of $A_{17}$ to That of $\mathbb{E}_{7}$}

We will freely use notations and results in string algebra approach as in $[\mathbb{E} 1, \mathbb{E} 2, \mathbb{K a l}, \mathbb{K a 2}, \mathbb{O} 1, \mathbb{O} 2, \mathbb{O} 3$, Sul. We first look at the subalgebra generated by the Jones projections in the string algebra of $E_{7}$ because the Jones projections are always flat. Then we get a commuting square 
embedding of two string algebras as in [GHD, §4.5], and the graphs for embeddings are obtained as in $[\mathbb{E} \mathbb{G} 1]$, [EG2], [OKK $]$ or $[\mathbb{Z} \mathbb{Z}$, page 622]. We label the vertices of the Coxeter-Dynkin diagram $\mathbb{E}_{7}$ as follows.

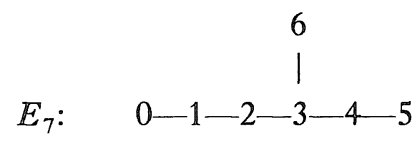

Because we already know that the connections on $E_{7}$ are not flat, $N^{\prime} \cap M_{k}, k \leq 6$, are generated by the Jones projections. So we look at the level $k=7$. If we look at the minimal projection orthogonal to the Jones projections in $N^{\prime} \cap M_{7}$ and check its string algebra expression in the $\mathbb{E}_{7}$ string algebra, we know that it is a sum of three minimal projections and that they are in summands corresponding to the vertices $0,2,6$ respectively. (We use the preceding embedding graphs for this.) Because this projection is flat, we cut the string algebras making $M_{7}$ by this projection and get the following commuting square series, and proceed as in [12].

$$
\begin{aligned}
& B_{00} \subset B_{01} \subset B_{02} \subset \cdots \\
& \cap \cap \cap \\
& B_{10} \subset B_{11} \subset B_{12} \subset \cdots
\end{aligned}
$$

Here $B_{00} \subset B_{01} \subset \cdots$ is the series of string algebras of $E_{7}$ starting from the vertex 0 and $B_{10} \subset B_{11} \subset \cdots$ is the series of string algebras of $E_{7}$ starting
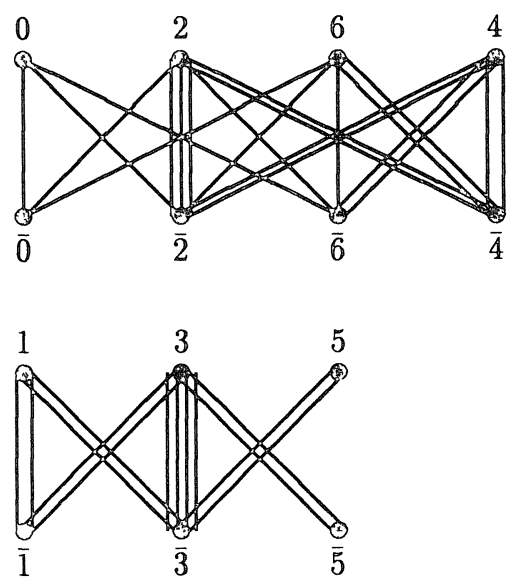

Figure 1.1 
from three vertices $0,2,6$. Next we compute the two vertical embedding graphs of (1.1). This is done just by counting the dimensions in the two Bratteli diagrams, and we get the following two graphs as in Figure 1.1, where we used notations $\overline{0}, \overline{1}, \ldots, \overline{6}$ for denoting the vertices of $E_{7}$ for the string algebras $B_{10} \subset B_{11} \subset \cdots$ as in [I2].

\section{§2. Computations of Connection Matrices}

Next we look at the series (1.1) and will determine the connection for this series just by studying the graphs, or at least we compute several entries of the connection matrices in a way similar to that in [12] with the same notation as in [12]. Of course, there is a connection for the series (1.1) arising from the original connection, but it is very hard to compute that explicitly. We will determine the information that we need regarding the connection from the graphs using gauge choices without considering the original connection $E_{7}$. This was the basic idea of Izumi [12]. We do not have to determine all the entries completely, so we use the symbol * for entries which we do not determine. Our aim in this section is to prove that after certain gauge choices all the connection entries are given by the following list.
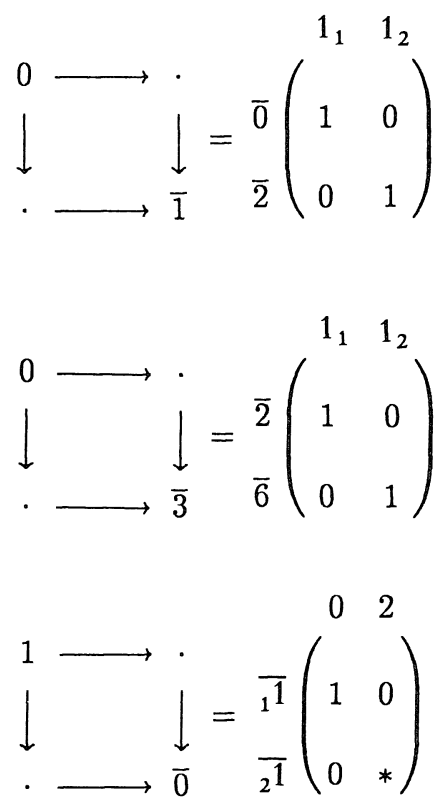
(2.4)

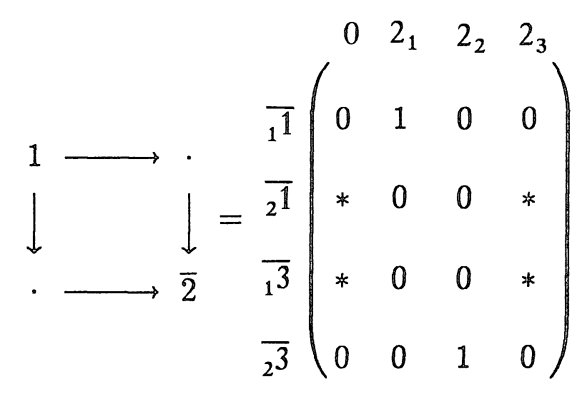

$(2.5)$

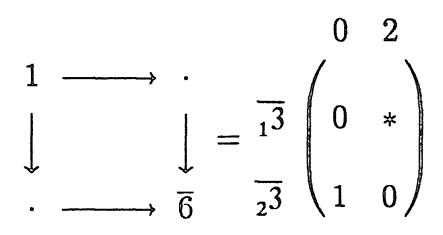

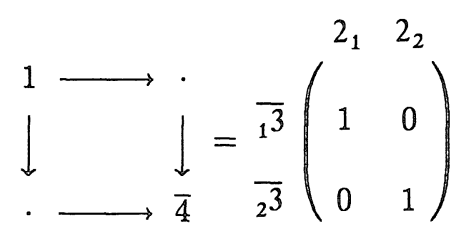

(2.7)

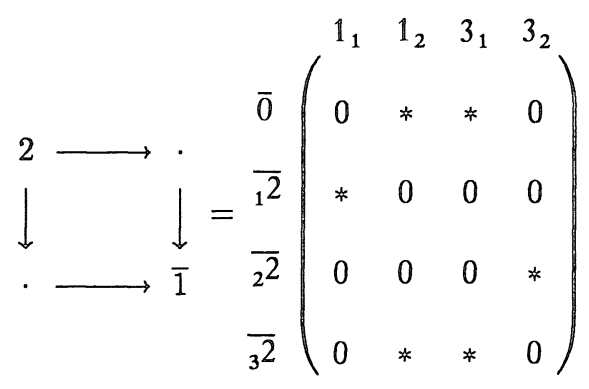


(2.8)

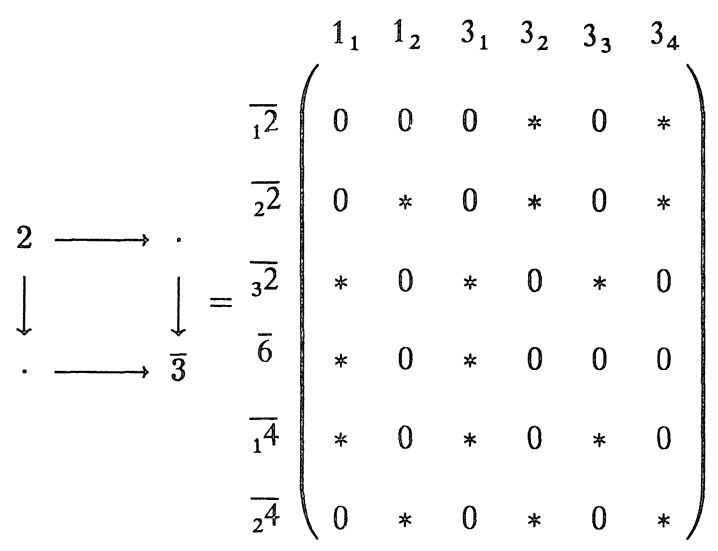

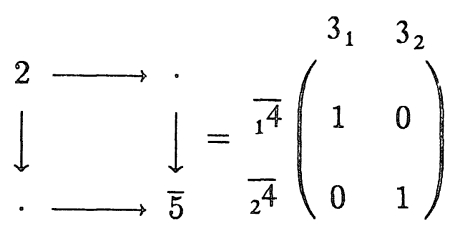

(2.10)

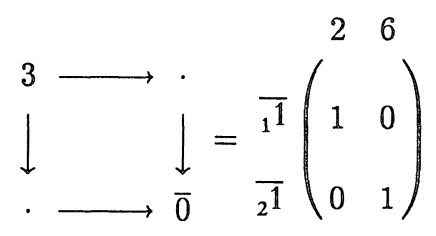

(2.11)

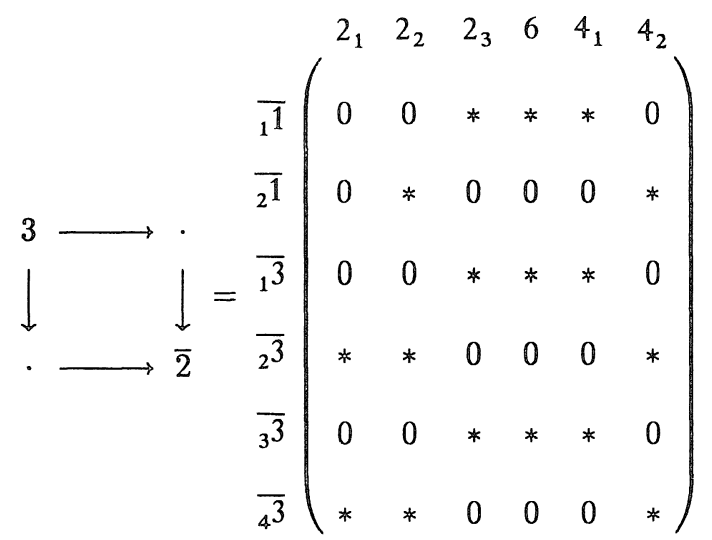




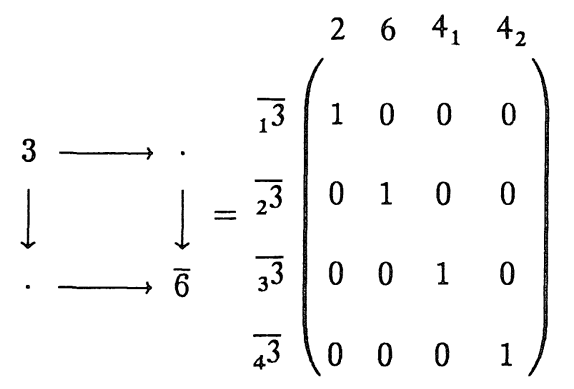

(2.13)

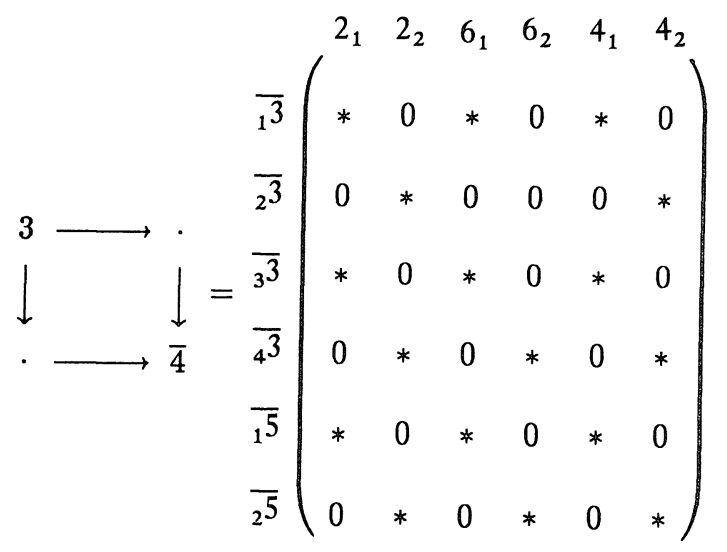

(2.14)

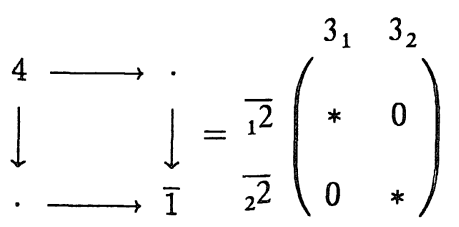

(2.15)

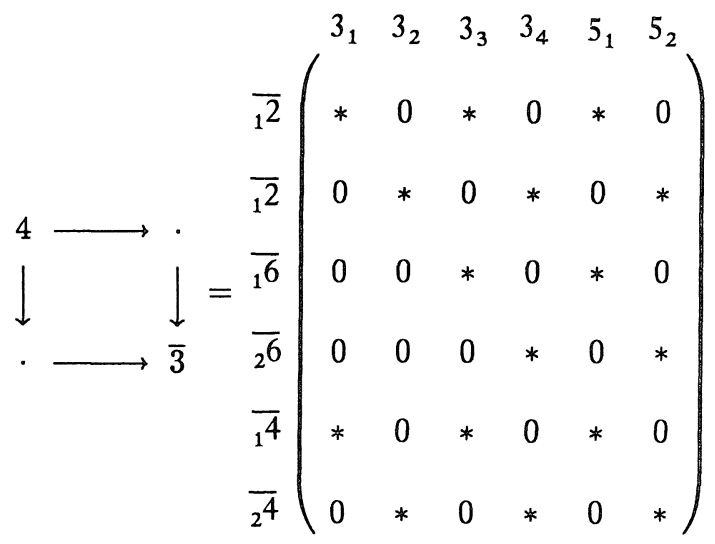




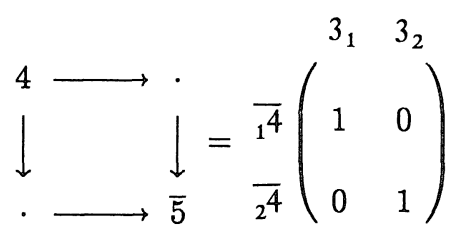

(2.17)

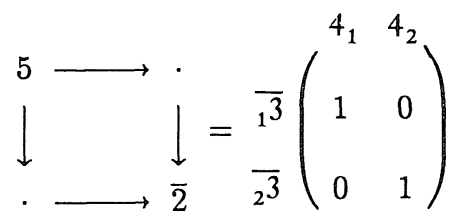

(2.18)

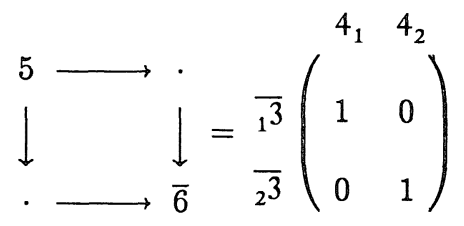

(2.19)

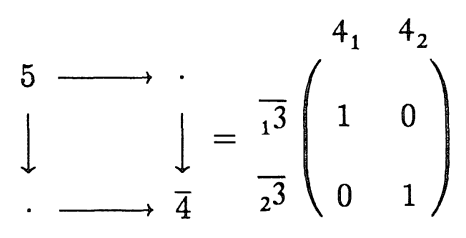

$(2.20)$

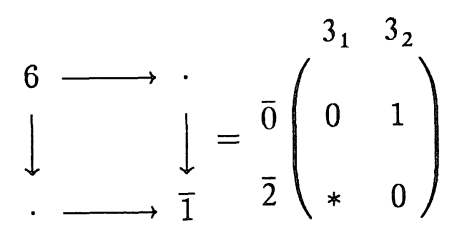

(2.21)

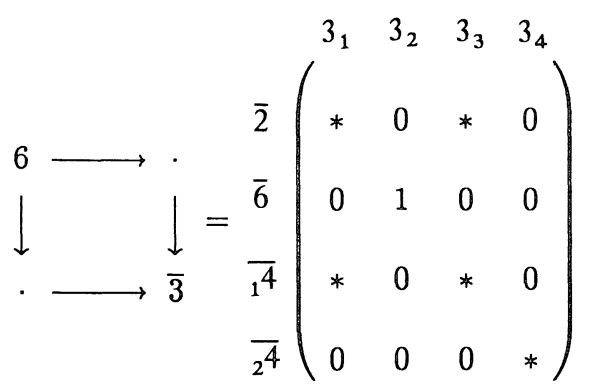




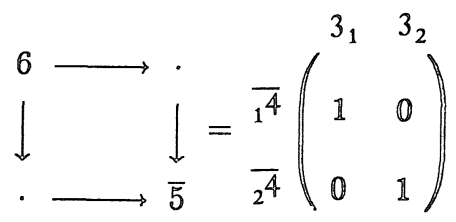

We now prove the above claim. In the first place, we make gauge choices for the following pairs of vertices in this order:

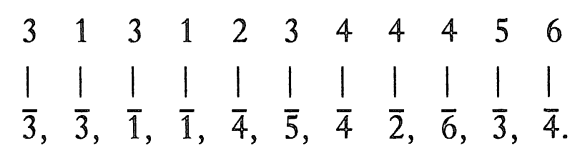

In this way, we fix the matrices (2.12), (2.2), (2.10), (2.1), (2.6), (2.9), (2.16), (2.17), (2.18), (2.19), and (2.22) as desired.

By the crossing symmetry

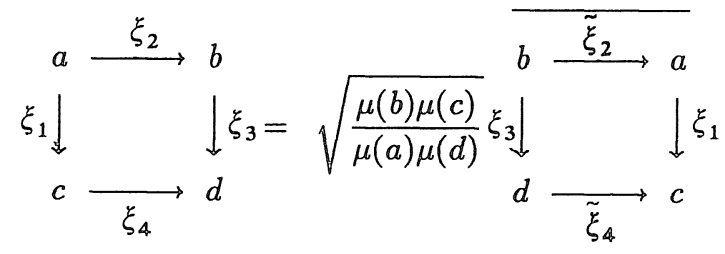

and (2.1), (2.2), we can partially fill entries of (2.4). Then by a gauge choice

for $\overline{2}$, we get (2.4) as desired.

Using the crossing symmetry, (2.10), and $\frac{\mu(6) \mu(1)}{\mu(3) \mu(0)}=1$, we get (2.20). Similarly, using the crossing symmetry, (2.2), and $\frac{\mu(6) \mu(1)}{\mu(3) \mu(0)}=1$, we get (2.5). Similarly, using the crossing symmetry and (2.1), we get (2.3).

Next we fill several entries from the determined entries and the crossing symmetry. Using unitarity, we get (2.7) as above. After filling further entries with the crossing symmetry, we get the following expression for (2.15). 


\begin{tabular}{|c|c|c|c|c|c|c|}
\hline & $3_{1}$ & $3_{2}$ & $3_{3}$ & $3_{4}$ & $5_{1}$ & $5_{2}$ \\
\hline$\overline{2}$ & $-\sqrt{\frac{\mu(2)}{\mu(4)}} a$ & $-\sqrt{\frac{\mu(2)}{\mu(4)}} \bar{b} \omega$ & $-\sqrt{\frac{\mu(5)^{2} \mu(2)}{\mu(3)^{2} \mu(4)}}$ & 0 & $\sqrt{\frac{\mu(5) \mu(2)}{\mu(3) \mu(4)}}$ & 0 \\
\hline$\overline{2}$ & $-\sqrt{\frac{\mu(2)}{\mu(4)}} b$ & $\sqrt{\frac{\mu(2)}{\mu(4)}} \bar{a} \omega$ & 0 & $-\sqrt{\frac{\mu(5)^{2} \mu(2)}{\mu(3)^{2} \mu(4)}}$ & 0 & $\sqrt{\frac{\mu(5) \mu(2)}{\mu(3) \mu(4)}}$ \\
\hline$\overline{6}$ & 0 & 0 & $\sqrt{\frac{\mu(6)}{\mu(4)}}$ & 0 & $\sqrt{\frac{\mu(5) \mu(6)}{\mu(3) \mu(4)}}$ & 0 \\
\hline & 0 & 0 & 0 & $\sqrt{\frac{\mu(6)}{\mu(4)}}$ & 0 & $\sqrt{\frac{\mu(5) \mu(6)}{\mu(3) \mu(4)}}$ \\
\hline & $a$ & $\bar{b} \omega$ & $-\frac{\mu(5)}{\mu(3)}$ & 0 & $\sqrt{\frac{\mu(5)}{\mu(3)}}$ & 0 \\
\hline & $b$ & $-\bar{a} \omega$ & 0 & $-\frac{\mu(5)}{\mu(3)}$ & 0 & $\sqrt{\frac{\mu(5)}{\mu(3)}}$ \\
\hline
\end{tabular}

where $|\omega|=1$, and $a, b, \omega \in \mathbb{C}$.

Using this, first we get the following expression for (2.13).

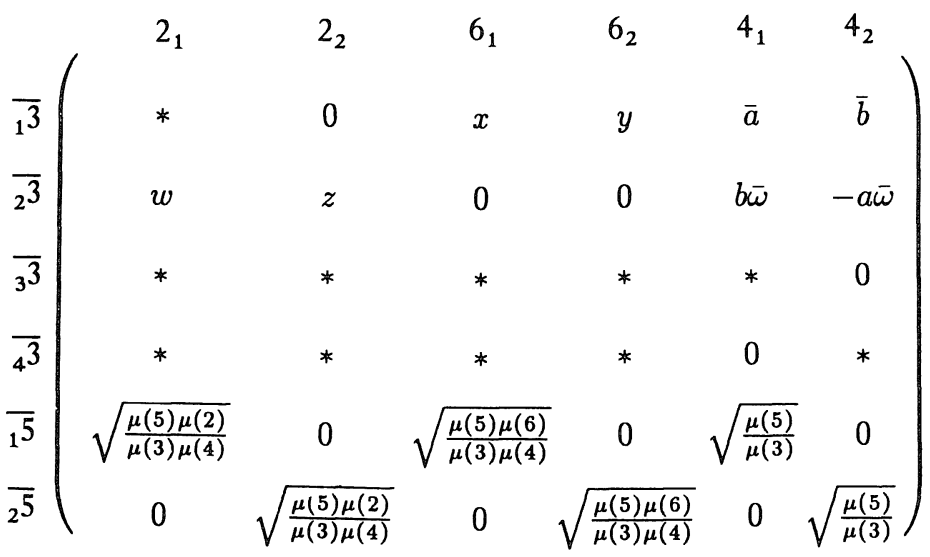

where $x, y, z, w \in C$. Using the orthogonality of the first and second rows and the fact that the $(1,1)$-entry is not zero, which comes from (2.8), we can conclude that $w=0$. Then by the orthogonality of the second and the fifth rows, we can conclude that $b=0$. By the orthogonality of the first and sixth rows, we next get $y=0$. Then (2.15) is of the desired form. In (2.8), the entry $\left(\overline{1}, 33_{2}\right)$ is 0 , and this implies that the entry $\left(\overline{3^{2}}, 3_{2}\right)$ is 0 by orthogonality of the first and the fourth columns. Then the orthogonality 
relations give (2.11) in the desired form. Finally we get (2.14), (2.8), (2.13), and (2.21) in this order, and we are done.

\section{§3. Computing the Flat Part and the Goodman- de la Harpe-Jones Subfactor for $A_{17}$ and $E_{7}$}

We look at the following diagram for computing the flat part.

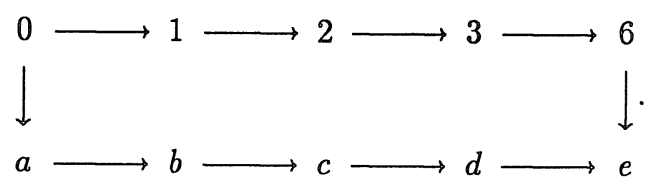

For each choice of vertex $e$, we determine the vertex $a$ for which the above diagram can have a non-zero value. This is done as in [12] with the matrices in $\S 2$, and we get the following.

(1) If $e$ is $\overline{0}$, then $a$ is $\overline{6}$.

(2) If $e$ is $\overline{2}$, then $a$ is $\overline{2}$.

(3) If $e$ is $\overline{6}$, then $a$ is $\overline{0}$ or $\overline{6}$.

(4) If $e$ is $\overline{4}$ and the vertical edge from 6 to 4 is labeled as 1 , then $a$ is $\overline{2}$.

(5) If $e$ is $\overline{4}$ and the vertical edge from 6 to 4 is labeled as 2 , then $a$ is $\overline{0}$ or $\overline{6}$.

This shows that the vertical string $(0 \rightarrow \overline{2}, 0 \rightarrow \overline{2})$ commutes with all the horizontal strings on $E_{7}$ starting from the vertex 0 , because the horizontal string algebras are generated by the Jones projections and a single projection $(0 \rightarrow 1 \rightarrow 2 \rightarrow 3 \rightarrow 6, \quad 0 \rightarrow 1 \rightarrow 2 \rightarrow 3 \rightarrow 6)$. This means that the higher relative commutants arising from the $E_{7}$ connections are strictly larger than the string algebras of $A_{17}$. We have thus proved that the principal graph of the subfactors arising from the $E_{7}$ connections is $D_{10}$ as desired. Note that there are two (mutually non-equivalent) connections on the graph $E_{7}$, but both give the same subfactor, since the subfactor with the principal graph $D_{10}$ is unique.

In general, we have the following proposition for the flat parts.

Proposition 3.1. Suppose we have a double complex $\left(A_{k l}\right)$ of string algebras arising from four graphs as in [03, Ka2]. Set the flat parts $B_{k}=A_{k, \infty} \cap A_{0, \infty}^{\prime}$. Then the following is a series of commuting squares of period 2 . 


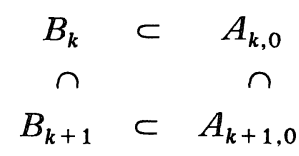

Proof. Let $e_{k}$ be the $k$-th vertical Jones projection in $A_{k+1,0}$. Then for $x \in B_{k+1}$, we get $e_{k} x e_{k}=E_{B_{k}}(x) e_{k}=E_{A_{k}}(x) e_{k}$, hence $E_{B_{k}}(x)=E_{A_{k}}(x)$. This gives the conclusion.

Q.E.D.

From this general statement and the above computation, we can conclude existence of a commuting square which embeds the string algebra of $D_{10}$ from $*$ to the string algebra of $E_{7}$ from $*$. By direct computations, we can see that the two graphs for embedding are also $D_{10}$ and $E_{7}$ respectively in this case.

Goodman, de la Harpe, and Jones [GHJ, §4.5] constructed subfactors from commuting squares embedding the string algebras of $A_{n}$ from $*$ to the string algebras of other $A-D-E$ graphs, and Okamoto [Ok] computed the principal graphs of these subfactors. In the case of $E_{7}$ with the choice of * as the starting vertex, the above result shows there is an intermediate subfactor for the Goodman-de la Harpe-Jones subfactors. Their numerical index value [GHJ, Proposition 4.5.2] for this subfactor is really $8 \cos ^{2} \frac{\pi}{18}$, and the above intermediate subfactor splits this number as $2 \times 4 \cos ^{2} \frac{\pi}{18}$. Existence of such an intermediate subfactor can be also seen from the principal graph of Okamoto [OK, DZ1] and a result of Pimsner-Popa on existence of normalizers in [PP, Proposition 1.7].

\section{$\S 4$. Comments on Conformal Field Theory}

We now discuss meaning of the above computations from the viewpoint of conformal field theory. These observations were bases of Zuber's conjecture mentioned in the introduction.

In the $A-D-E$ classification of $[\mathbf{C I Z}, \mathbf{K t}]$ on level $k S U(2) W Z W$-models, the $A_{n}, D_{2 n}, E_{6}, E_{8}$ models have block-diagonal modular invariants and the $D_{2 n+1}$ and $E_{7}$ models have off-diagonal modular invariants. Comparing this with the $A-D-E$ classification of subfactors announced by $\mathrm{A}$. Ocneanu, we easily notice a rather mysterious similarity between block diagonal modular 
invariants and flat connections. Furthermore, this similarity still holds between our work on orbifold subfactors from Hecke algebras [ERI] and modular invariants of the orbifold graphs $\mathscr{D}^{(n)}[\mathrm{DZ} 2$, Tables $\mathbb{I}, \mathbb{I} \mathbb{I}]$.

Dijkgraaf-Verlinde [DV] studied the relation between off-diagonal modular invariants and non-trivial automorphism of the fusion algebra of conformal field theory. They further discussed the following $E_{7}$ pairing in [DV, pages 95-96]. The partition functions labeled by $D_{10}$ and $E_{7}$ are given as follows.

$$
\begin{aligned}
D_{10}: & Z=\left|\chi_{1}+\chi_{17}\right|^{2}+\left|\chi_{3}+\chi_{15}\right|^{2}+\left|\chi_{5}+\chi_{13}\right|^{2}+\left|\chi_{7}+\chi_{11}\right|^{2}+2\left|\chi_{9}\right|^{2} \\
E_{7}: & Z=\left|\chi_{1}+\chi_{17}\right|^{2}+\left|\chi_{5}+\chi_{13}\right|^{2}+\left|\chi_{7}+\chi_{11}\right|^{2}+\left|\chi_{9}\right|^{2}+\left[\left(\chi_{3}+\chi_{15}\right) \bar{\chi}_{9}+\text { c.c. }\right] .
\end{aligned}
$$

Both have the same chiral algebra containing the $S U(2)_{k=16} \mathrm{KM}$-algebra and the characters are given by $\chi_{1}+\chi_{17}, \chi_{3}+\chi_{15}, \chi_{5}+\chi_{13}, \chi_{7}+\chi_{11}, \chi_{9}^{+}$, and $\chi_{9}^{-}$. Then the corresponding operators are denoted as $1, \varphi_{1}, \varphi_{2}, \varphi_{3}, \varphi_{4}^{+}$ and $\varphi_{4}^{-}$. In this setting, the off-diagonal pairing is given by the exchange of $\varphi_{1}$ and $\varphi_{4}^{+}$. They also computed the same fusion rules of these models with $S$-matrix and observed that the pairing exchanging $\varphi_{1}$ and $\varphi_{4}^{+}$gives a $\mathbb{Z}_{2}$ symmetry of the fusion rules. Or, in the notation of [ $\mathbb{Z}$, page 315 , Table II], this automorphism of order 2 exchanges the block with exponents $(3,15)$ with the one of exponent 9. (This fusion rule is the same as the one obtained with the $N-N$ bimodules arising from the subfactor with $D_{10}$ principal graph. See [II] for fusion rule computations.) With this symmetry, one can twist the characters $\chi$ with respect to the conjugated characters $\bar{\chi}$ and get the $E_{7}$ invariant which is not block diagonal from the $D_{10}$ invariant which is block diagonal. With this observation and similarity between block diagonal modular invariants and flat connection, Zuber conjectured that the (non-flat) connections on the $E_{7}$ graph would give principal graph $D_{10}$. Our computations proved this conjecture and gave a further evidence on this similarity. Our commuting square obtained at the end of $\S 4$ clarifies a relation between the two diagrams suggested by this pairing.

\section{References}

[ABF] Andrews, G.E., Baxter, R.J. and Forrester, P.J., Eight vertex SOS model and generalized Rogers-Ramanujan type identities, J. Stat. Phys., 35 (1984), 193-266.

[Ba] Baxter, R.J., Exactly solved models in statistical mechanics, Academic Press, New York, 1982. 
[BN] Bion-Nadal, J., An example of a subfactor of the hyperfinite $I I_{1}$ factor whose principal graph invariant is the Coxeter graph $E_{6}$, in Current Topics in Operator Algebras, World Scientific Publishing, (1991), 104-113.

[BG] de Boer, J. and Goeree, J., Markov traces and $\mathrm{II}_{1}$ factors in conformal field theory, Comm. Math. Phys., 139 (1991), 267-304.

[CIZ] Cappelli, A., Itzykson, C. and Zuber, J.-B., The $A-D-E$ classification of minimal and $A_{1}^{(1)}$ conformal invariant theories, Comm. Math. Phys., 113 (1987), 1-26.

[DJMO] Date, E., Jimbo, M., Miwa, T. and Okado, M., Solvable lattice models, in Theta functions-Bowdoin 1987, Part 1, Proc. Sympos. Pure Math. 49, Amer. Math. Soc., Providence, R.I., pp. 295-332.

[DJN] Durhuus, B., Jakobsen, H.P. and Nest, R., Topological quantum field theories from generalized $6 j$-symbols, preprint.

[DZ1] Di Francesco, P. and Zuber, J.-B., $S U(N)$ lattice integrable models associated with graphs, Nucl. Phys., B338 (1990), 602-646.

[DZ2] — $S U(N)$ lattice integrable models and modular invariance, to appear in Proc. Trieste Conf. Recent Developments in Conformal Field Theories, Trieste, 1989.

[DV] Dijkgraaf, R. and Verlinde, E., Modular invariance and the fusion algebra, Proceedings of the Annecy Conference on Conformal Field Theory, Nucl. Phys. B, Proc. Suppl., 5B (1988), 87-97.

[DHVM] Dixon, L., Harvey, J.A., Vafa, C. and Witten, E. Strings on orbifolds, Nucl. Phys., B261 (1985), 678-686, B274 (1986), 285-314.

[E1] Evans, D.E., The $C^{*}$-algebras of topological Markov chains, Tokyo Metropolitan University Lecture Notes, 1984.

[E2] - Quasi-product states on $C^{*}$-algebras, in Operator algebras and their connections with topology and ergodic theory, Springer Lect. Notes in Math., 1132 (1985), 129-151.

[EG1] Evans, D.E. and Gould, J.D., Dimension groups and embeddings of graph algebras, to appear in Internat. J. Math.

[EG2] - Presentations of AF algebras associated to T-graphs, to appear in Publ. RIMS, Kyoto Univ.

[EK1] Evans, D.E. and Kawahigashi, Y., Orbifold subfactors from Hecke algebras, to appear in Comm. Math. Phys.

[EK2] - From subfactors to 3-dimensional topological quantum field theories and back, to appear in Astérisque.

[F] Fendley, P., New exactly solvable orbifold models, J. Phys., A22 (1989), 4633-4642.

[FG] Fendley, P. and Ginsparg, P., Non-critical orbifolds, Nucl. Phys., B324 (1989), 549-580.

[GHJ] Goodman, F., de la Harpe, P. and Jones, V.F.R., Coxeter graphs and towers of algebras, MSRI publications 14, Springer, 1989.

[I1] Izumi, M., Application of fusion rules to classification of subfactors, Publ. RIMS, Kyoto Univ., 27 (1991), 953-994.

[I2] - On flatness of the Coxeter graph $E_{8}$, to appear in Pac. J. Math.

[IK] Izumi, M. and Kawahigashi, Y., Classification of subfactors with the principal graph $D_{n}^{(1)}$, J. Funct. Anal., 112 (1993), 257-286.

[Ji] Jimbo, M.(editor), Yang-Baxter equation in integrable systems, Adv. Ser. Math. Phys. 10, World Scientific, 1989.

[Jo] Jones, V.F.R., Index for subfactors, Invent. Math., 72 (1983), 1-15.

[Kt] Kato, A., Classification of modular invariant partition functions in two dimensions, Mod. Phys. Lett., A2 (1987), 585-600.

[Kal] Kawahigashi, Y., On flatness of Ocneanu's connections on the Dynkin diagrams and classification of subfactors, to appear in J. Funct. Anal.

[Ka2] - Exactly solvable orbifold models and subfactors, in Functional Analysis and Related Topics, Lect. Notes in Math. 1540, Springer Verlag, 1992. 
[Ko] Kostov I., Free field presentation of the $A_{n}$ coset models on the torus, Nucl. Phys., B300 (1988), 559-587.

[O1] Ocneanu, A., Quantized group string algebras and Galois theory for algebras, in Operator algebras and applications, 2, Warwick, 1987, London Math. Soc. Lect. Note Series 136, Cambridg University Press, (1988) 119-172.

[O2] - Graph geometry, quantized groups and nonamenable subfactors, Lake Tahoe Lectures, June-July, 1989.

[O3] - Quantum symmetry, differential geometry of finite graphs and classification of subfactors, University of Tokyo Seminary Notes 45, Notes recorded by Y. Kawahigashi, 1991.

[O4] Ocneanu, A., An invariant coupling between 3-manifolds and subfactors, with connections to topological and conformal quantum field theory, unpublished announcement, 1991.

[Ok] Okamoto, S., Invariants for subfactors arising from Coxeter graphs, in Current Topics in Operator Algebras, World Scientific Publishing, (1991), 84-103.

[Pa] Pasquier, V., Two-dimensional critical systems labelled by Dynkin diagrams, Nucl. Phys., B285 (1987), 162-172.

[PP] Pimsner, M. and Popa, S., Entropy and index for subfactors, Ann. Scient. Éc. Norm Sup., 19 (1986), 57-106.

[P1] Popa, S., Orthogonal pairs of *-subalgebras in finite von Neumann algebras, J. Operator Theory, 9 (1983), 253-268.

[P2] - Classification of subfactors: reduction to commuting squares, Invent. Math., 101 (1990), 19-43.

[P3] Popa, S., Classification of amenable subfactors of type II, to appear in Acta Math.

[R] Roche, Ph., Ocneanu cell calculus and integrable lattice models, Comm. Math. Phys., 127 (1990), 395-424.

[Sc] Schou, J., Commuting squares and index for subfactors, $\mathrm{Ph}$. D. Thesis, Odense University, 1990.

[So] Sochen, N., Integrable models through representations of the Hecke algebra, Nucl. Phys., B360 (1991), 613-640.

[Su] Sunder, V.S., A model for AF-algebras and a representation of the Jones projections, J. Operator Theory, 18 (1987), 289-301.

[SV] Sunder, V.S. and Vijayarajan, A.K., On the non-occurrence of the Coxeter graphs $\beta_{2 n+1}, E_{7}, \mathrm{D}_{2 n+1}$ as principal graphs of an inclusion of $I I_{1}$ factors, to appear in Pac. J. Math.

[TV] Turaev, V.G. and Viro, O.Y., State sum invariants of 3-manifolds and quantum 6j-symbols, Topology, 31 (1992), 865-902.

[W] Witten, E., Gauge theories and integrable lattice models, Nucl. Phys., B322 (1989), 629-697

[Z] Zuber, J.-B., Graphs, algebras, conformal field theories and integrable lattice models, Nucl. Phys. B(Proc. Suppl.), 18B (1990), 313-326. 